International Journal of Advanced Trends in Computer Science and Engineering

Available Online at http://www.warse.org/IJATCSE/static/pdf/file/ijatcse55922020.pdf

https://doi.org/10.30534/ijatcse/2020/55922020

\title{
Efficient and Accurate Hybrid Deep Learning Model for Multimodal Disease Risk Prediction
}

\author{
A Veena Reddy ${ }^{1}$, Hari Kiran Vege ${ }^{2}$, Kolla Bhanu Prakash ${ }^{3}$ \\ ${ }^{1,2,3}$ Department of Computer Science and Engineering, K. L. Deemed to be University \\ 3drkbp@kluniversity.in
}

\begin{abstract}
Biomedical and restorative organizations and correct examination of supportive information play a key role in early illness recognizing which currently depends on the use of Big Data. Without proper supportive information, we cannot expect examination accuracy. In chronicle diseases such as diabetes, hypertension, cerebral infarction and asthma the forecast of infection over the genuine information is been gathered from the hospital. We can foresee a change in the big data particularly in the existing new "Convolutional Neural Network-based Multimodal Disease Risk Prediction" (CNN-MDRP) estimation which utilizes structured and unstructured data. The slightest data reduction leads to precision reduction. It will clear the defilement of information by Genetic Algorithm. The unstructured information will be changed over into the structured data with the help of Recurrent Neural Network (RNN).
\end{abstract}

Keywords: Big Data, Convolutional Neural Network, Data Reduction, Genetic Algorithm, Recurrent Neural Network

\section{I.INTRODUCTION}

As stated by a survey that more than 17.7 million passing's happened all over the world in a year is due to heart-related problems. The cardiovascular problem would a major problem among the majority common problem of the updated world. A heart attack is one of the major deadly problems that it knocks at any point without intimation and silently kills a person which is occurred frequently and predicted by the doctors more frequently. Here is an estimated study says that 7.4 million deaths are due to coronary heart disease and 6.7 million deaths are due to heart strokes this is due to lack of specialists and day by day increasing wrong diagnosed cases that need a building system that could predict cardiovascular diseases. So here we introduce a new open-source code in research and also in developing new methods using medical data mining and machine learning techniques [1], [3]. The main objective of this research work is to use classification algorithms for identifying the key patterns and features of silent heart attack diagnosis and then select a most relevant attribute for that problem. To enhance the result accuracy we utilize Recurrent Neural Network but implementing such a system is not unique and also the present system has drawbacks for finding out the probabilities of silent heart attacks. The present paper proposes the implementation of novel features in developing inclusive systems.

\section{LITERATURE REVIEW}

The hazardous disease is being anticipated through a new Convolution Neural Network-based Multimodal Disease Risk Prediction (CNN-MDRP) which is a recurring formation to capture contextual information [2]. The new deep learning architecture Bi-CNN-MI Paraphrase Identification (PI) is the PI that thinks about two sentences on numerous levels of granularity and reword generally has the same meaning. The parameters of all the models are upgraded for PI. Utilization of dialect displaying assignment is to tackle the absence of preparing information. Here they analyze machine learning algorithms like Decision Tree, Bayes algorithm, Support Vector Machine (SVM) and Nearest Neighbor. Present algorithms utilize for the most part. They are used for the prediction of bunch enrollment for information examples. They present a comparative assessment of different algorithms. In EHR and the mining of it, an expansive scope of moral, legitimate and specialized reasons may prevent the methodical testimony. In data mining, they extricate the concealed prescient information from the expansive database. The capability of Electronic Health Record (EHR) [4] is for setting up new patients by uncovering the obscure disease correlation. The telehealth administrations are being utilized which are known as the telehealth warning administrations. They are for the most part utilized as a part of metropolitan cities. Due to telehealth administrations, the patients can get assistance easily. A fast incremental in the telehealth framework [5] has gotten different methods like cloud computing and big data. The dynamic programming creates ideal arrangements to take care of information sharing systems. In this, it thinks about the transmission probabilities, the planning imperatives, and augmenting the system limits. For a text sentiment analysis 
with jointed Convolutional Neural Network (CNN) and Recurrent Neural Network (RNN) architecture [6], taking the upsides of both like coarse-grained local features highlights which are created by CNN and long-distance dependencies learned through the RNN. It employs a max-pooling layer that automatically judges, which words to play on. The regional chronic disease has been focused on. Attention has been paid on both structured and unstructured data.

The regional chronic disease has been focused on. Attention has been paid on both structured and unstructured data. It employs a max-pooling layer that automatically judges, which words play an important role in text classification to capture the key components in texts [22]. The information contains in traits with missing information esteems are critical in enhancing decision - making procedure of an association. For a text sentiment analysis with jointed Convolutional Neural Network (CNN) and Recurrent Neural Network (RNN) architecture [7], taking the upsides of both like coarse-grained local features highlights which are created by $\mathrm{CNN}$ and long-distance dependencies learned through the RNN [9] [20]. The learning process on each occurrence is vital as it might contain a few uncommon learning. There are different techniques to deal with missing information in decision tree learning. The proposed ascription calculation is based on the hereditary calculation that utilizes space esteems for that property like a pool of arrangements. Survival of the fittest is the premise of genetic algorithm. The wellness work is grouping exactness of an occasion with credited an incentive on the choice tree.

The worldwide hunt system utilized as a part of a genetic algorithm [26] is relied upon to get an optimal solution. Our strategy viably fused area learning about the restorative characterization of both disease and EHRs into an information-driven approach [8]. Exploratory outcomes on a genuine dataset from a healing facility exhibited the viability of our proposed strategy. Their approach consolidated undertaking relatedness, i.e., how every illness relates with others, in a suitable way, which prompted a change in the prescient execution [10]. The joining of area learning about the therapeutic order of EHRs was moreover powerful. Besides, the after-effects of the investigations of the diseasespecific prescient highlights not just contained discoveries steady with existing medicinal area learning, yet additionally delivered a few theoretical proposals. To upgrade an understanding of disease particular settings and additionally to enhance the prescient execution in mortality displaying in acute hospital care. Their technique and results could be powerful In it presents a straightforward, yet capable modification to the simple RNN (SRN) [12] architecture, the Clockwork RNN (CW-RNN) [14], in which the covered up layer is divided into partitioned modules, each handling contributions at its particular worldly granularity, making algorithms just at its recommended clock rate. As against making the standard RNN models more minds boggling, CW-RNN reduces the number of SRN parameters, enhances the execution fundamentally in the undertakings tried, and accelerates the network evaluation. The system is illustrated in preparatory trials including three errands: audio signal generation, TIMIT talked word classification [13], where it beats both SRN and LSTM systems, and internet penmanship recognition, [11] where it beats SRNs.

\section{IMPLEMENTATION}

As indicated by the writing survey, the AI methods utilized are pushing the precision until a specific breaking point. Anyway, the current frameworks utilized for a forecast of heart attack missing the mark regarding wanted precision in the outcomes. Besides, the issue with the present heart assault expectation framework is the employments of properties. The credits to be chosen for the expectation of heart assault are the customary ones and in this manner, the outcomes are creating incorrectly results many-a-times. The proposed model plans to separate the best possible properties from the datasets which will upgrade the accuracy of the expectation. It will likewise furnish the clients with a legitimate conclusion so the client comprehends the issue well absent a lot of trouble. The task separates itself by tackling the forces of both Deep learning and information mining [23]. The paper proposes a framework, with a solid forecast calculation, which executes incredible grouping ventures with a far-reaching reportage module. The undertaking the targets actualizing a self-learning convention with the end goal that the past contributions of the illness results decide the future conceivable outcomes of the heart ailment to a specific client. Various preparing sets will be utilized to make the forecast increasingly precise. Not exclusively does the datasets yet also besides the scribes to be utilized are chosen contemplating the different significant parameters and properties. The proposed model uses solid preprocessing devices to stay away from any mistakes identifying with the dataset in arrangement and expectation. Executing the before referenced model meets the objective of building up a framework with expanded precision of assessing the patient getting heart assault rate while tending to the disadvantages of the current framework The model which is proposed for Heart Attack Prediction System [15],[16] is made utilizing Deep learning algorithms [17] and approach. The proposed approach steps are listed in Figure 1.

\subsection{Data Source}

Dataset is an assortment of information questions that have quantities of properties which manage the fundamental qualities of an item. We got 303 records with 75 medicinal characteristics (factors) from the UCI Machine Learning Data source [24]. In the wake of preprocessing utilization of 270 records with 13 therapeutic characteristics. Out of these 13 traits, 7 have discrete qualities while 6 have persistent qualities. The Table 1 specifies the 13 characteristics which are used in our current approach for implementation. 


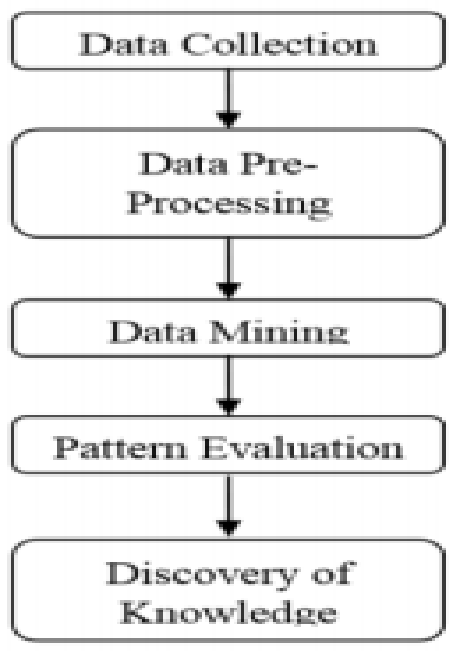

Figure 1: Steps Followed in Proposed Approach

\subsection{Recurrent Neural Network}

It is a model which cannot just learn neighborhood and worldly conditions in information yet also can suit variable succession lengths. This profound learning model has a basic structure with an inherent criticism circle enabling it to go about as an anticipating motor [25]. RNN is a customary neural system with an extra shrouded state where the concealed state impacts the neural system yield [18]. The shrouded state is refreshed on each information step. In RNN the yield of a layer is added to the following

Table 1: List of Characteristics Used for Implementation

\begin{tabular}{|c|c|c|}
\hline Name & Type & Description \\
\hline Age & Coetinucus & Age Age in yars \\
\hline Sex & Disertete & $0=$ femak $1=$ male \\
\hline$C_{p}$ & Discrete & $\begin{array}{l}\text { Chest puin type: } 1 \text { = typical angina, } 2 \text { = alypical } \\
\text { angina, } 3 \text { = non-anginal pain } 4 \text { asymptom }\end{array}$ \\
\hline Trestbps & Cotlinucus & Resing blood pressure (in mm $\mathrm{H}_{g}$ ) \\
\hline Chol & Continucus & Serum choleterol in mg(d) \\
\hline Fos & Discrate & Fasting blood sugan $\mid 20$ mold: 1-true 0-Falle \\
\hline $\begin{array}{l}\text { Exang Continucus Maximum } \\
\text { heart rade achieved }\end{array}$ & Discrete & Fxercise indused anginas. I = Yes $0=\mathrm{No}_{0}$ \\
\hline Talach & Coetinucess & Maximam heant rate achicved \\
\hline OAd peak ST & Coetinucus & Depression indexed by exercise relative to rest \\
\hline Slope & Discrete & $\begin{array}{l}\text { The skope of the peak exercise segmeat : } \\
1=\text { up sloping } 2=\text { flat } 3=\text { down sloping }\end{array}$ \\
\hline $\mathrm{Ca}$ & Cotinucus & $\begin{array}{l}\text { Number of major vessels colorod by flucroscopy } \\
\text { that ranged between } 0 \text { and } 3 \text {. }\end{array}$ \\
\hline Thal & Distete & $3=$ nermal 6 = fived defect $7=$ reversible defat \\
\hline Class & Distrete & $\begin{array}{l}\text { Dhagnosis classex } 0=\text { No Presence 1=Leas likely lo } \\
\text { have heart dsease } 2=>13 m>24=\text { More likely have } \\
\text { heart disease }\end{array}$ \\
\hline
\end{tabular}

info layer and encouraged go into a similar layer. Not at all like feed-forward systems [19], RNN can get the arrangement of qualities as information sources and it can create a succession of qualities as yields. However, in RNN issue of disappearing angle is more regrettable along these lines it prompts exponentially little slopes and rot of data through time.

\subsection{Algorithms}

CNN Algorithm:

1. The dataset may be changed over to the vector type.

2. After that expression embedding conveyed to adopt zero qualities that fill the information. That output of expression embedding to be convolution layer.

3. This Convectional layer made similarly as entered to pooling layer and we perform max pooling operation on conventional layer.

4. To max-pooling, those dataset change over-under settled length vector type. Pooling layer is joined for the full associated neural system.

5. The full association layer associated with that classifier that is softmax classifier.

Figure 2 architectural diagram of deep neural network training model [28] used in our implementation approach. Figure 3 provides the steps followed in our implementation approach.

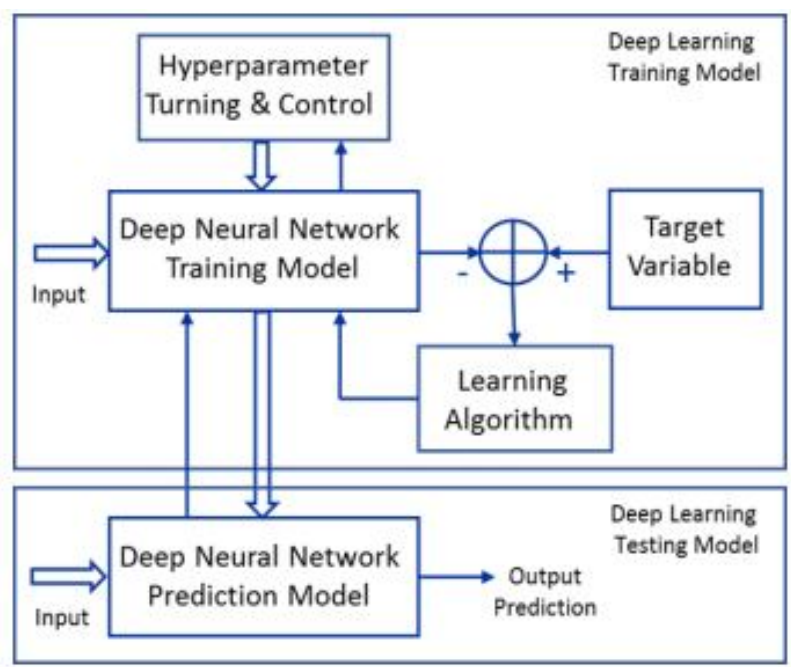

Figure 2: Deep Neural Network Model

RNN Algorithm:

We propose Toward RNN classifier similarly as the neural system group that might fuse diverse build classifiers under classifier ensembles models for arrangement issues. This project recommended that those effects about utilizing distinctive build classifiers once arrangement precision for RNN classifier group. Classifier ensembles with five build classifier need to be utilized on five restorative information sets. These come about assessed Also compared picking distinctive sort for choice tree algorithms for build classifier. The dependability about the arrangement for the greater part about datasets Furthermore classifier ensembles is expanded when we select those proper RNN classifiers accomplishes the base time needed on raise models. 
In general, RNN (Recurrent neural Network) may be choice tree built neural system would a group taking in strategy to classification, relapse Furthermore different tasks, that work Toward constructing An huge number about choice trees during preparation the long run And outputting those class that is those mode of the classes (classification) alternately mean prediction (regression) of the distinctive trees. RNN right for choice trees' propensity of In fitting should their preparation set RNN is An Factual algorithm [21] that is used to group focuses for information extraction [27] on practical bunches. The point when those information set will be substantial or there would a significant number variables it gets to be challenging will bunch the information on account of not all variables might a chance to be taken under account, thus the

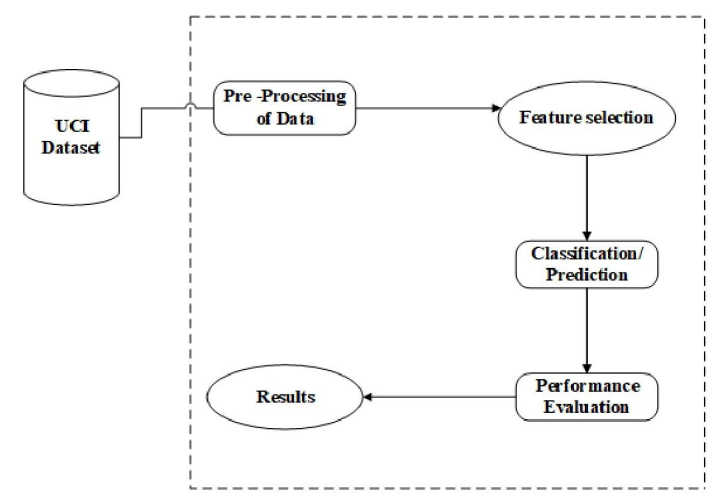

Figure 3: Steps Followed in Implementation Approach

calculation could additionally provide for a specific possibility that an information point belongs by An certain bunch. This may be how those groupings take spot. Those calculation groups the information (Stages 1, 2, 3 and 4) to gatherings Furthermore subgroups. If you might draw lines between those information focuses by a subgroup, furthermore lines that interface subgroups under aggregation and so forth. That structure might take a gander sort of in a tree. This is called a tree. During every part alternately hub in these cluster/tree variables would pick at arbitrary by the system to judge if information focuses bring an ended relationship or not a tree, variables are decided at arbitrary. After that whatever remains of that dataset (not the preparing set) may be used to anticipate which tree in the forests makes the best arrangement of the information focuses (in the dataset the good order is known). That tree with those the greater part predictive control may be demonstrated Likewise yield by that algorithm. RNN classifier algorithms [22] need aid in changing heulandites strategies. An RNN classifier wellness work is ascertained to find that RNN classifier approach. Previously, used those RNN classifier systems to those conjecture of breast tumor. This framework will be a mixture for those choice trees, ANN and logistic relapse. They utilized 699 records procured starting with those diabetics us patients toward the college of $\mathrm{w}$ is consigned. They used 9 pointer variables Furthermore 1 outcome variable for the majority of the data examination for 10-fold cross Regard. Those specialists declared that their RNN Classifier. Figure 4 gives clear picture of RNN classifier tree which is used in our implementation of the RNN algorithm.

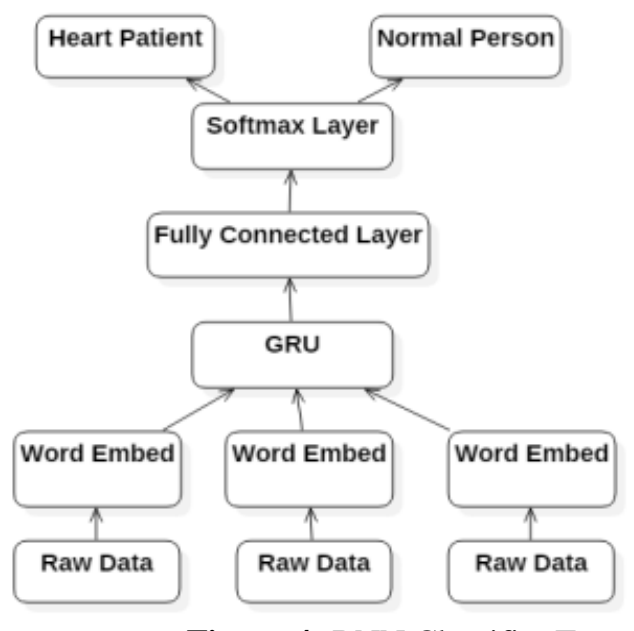

Figure 4: RNN Classifier Tree

\section{RESULTS AND DISCUSSION}

Figure 5 shows the features extraction from UCI data set total length of the features which are about 19. Figure 6 gives the neural network construction for the prediction. Figure 7 shows the epochs for the proposed RNN model. Figure 8 shows the model accuracy for the no of epochs and Figure 9 gives the model loss for the no of epochs

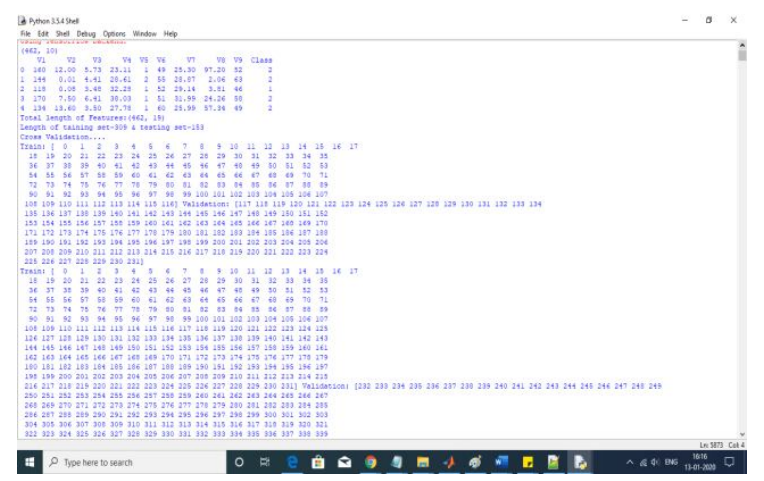

Figure 5: Features Extraction from UCI dataset

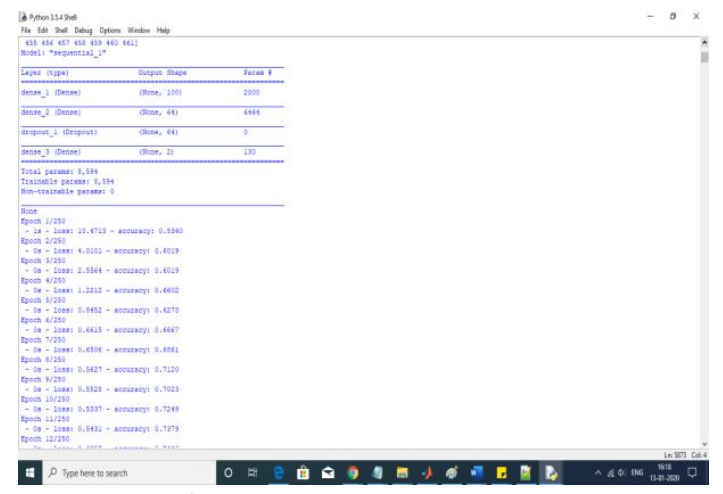

Figure 6: Neural Network Construction 


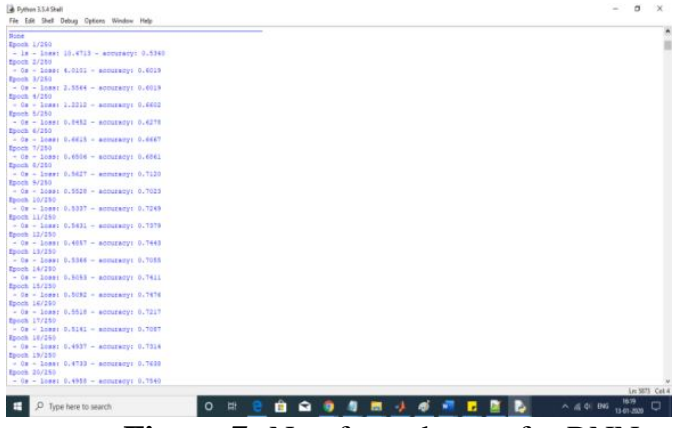

Figure 7: No of epochs run for RNN model.

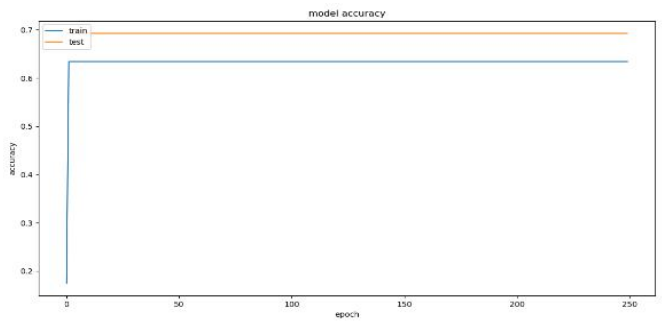

Figure 8: Model Accuracy for No of Epochs

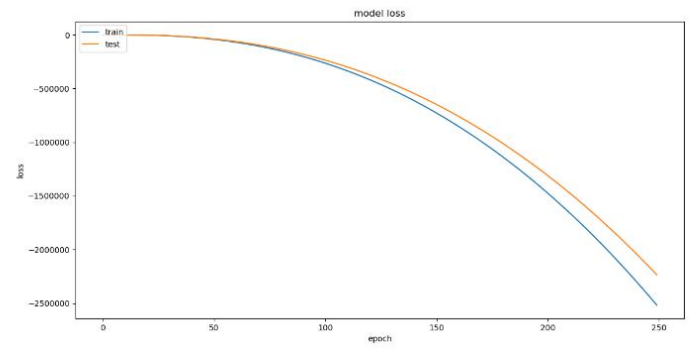

Figure 9: Model Loss for No of Epochs

RNN is executed previously; python and the streamlining utilizing Theano, a library on performing operations to a GPU. Information measurements were 13 to every quality Also 100 concealed layers were picked for the RNN. 150 records were utilized to preparing set Also 120 records were utilized for trying situated. Those model might have been prepared to 100 epochs and clump extent similarly as 10 and data might have been utilized Likewise the streamlining calculation. Toward each epoch, "Validation AUC" will a chance to be ascertained utilizing the acceptance set and assuming that it will be those best "Validation AUC" something like that far, those test set will be used to figure "Test AUC". The model for those best "Test AUC" might have been spared during that end of the preparing which originated to a chance to be $92 \%$. Table 2 lists the accuracy of different models it shows our current RNN model has high accuracy than any other model mentioned in the table.
Table 2: Techniques with their Accuracy

\begin{tabular}{|l|l|}
\hline \multicolumn{1}{|c|}{ TECHNIQUE USED } & \multicolumn{1}{c|}{ ACCURACY } \\
\hline CNN \& Decision Tree[5] & $82 \%$ \\
\hline SVM[6] & $84.12 \%$ \\
\hline Naïve Bayes[7] & $86.53 \%$ \\
\hline KSOM[8] & $88.9 \%$ \\
\hline C4.5 MAFIA K-means Cluster[9] & $89 \%$ \\
\hline DBN[10] & $90 \%$ \\
\hline RNN & $92 \%$ \\
\hline
\end{tabular}

\section{CONCLUSION}

After careful in-depth literature survey of Multimodal Disease Risk Prediction, it is observed that different Machine learning and deep learning algorithms lack required accuracy and efficiency. A standard algorithm which provides accurate results to quiet heart attack prediction was not available. Henceforth after careful analysis, we considered the perfect utilization of RNN and GRU. This system predicted heart attack to $92 \%$ and demonstrated an excellent source for foreseeing silent heart attack.

\section{REFERENCES}

1. Min Chen, Yixue Hao, Kai Hwang, Lu Wang, and Lin Wang,'Disease Prediction by Machine Learning over Big Data from Healthcare Communities." IEEE transaction, 2017, pp 88698879.

2. W. Yin and H. Schutze, "Convolutional neural network for paraphrase identification", in HLTNAACL, 2015, pp. 901-911.

3. Seema Sharma, Jitendra Agarwal, Shikha Agarwal, Sanjeev Sharma, "Machine Learning Techniques for Data Mining: A Survey", in Computational Intelligence and Computing Research, IEEE International Conference on. IEEE, 2013, pp.1-6.

4. Jensen PB, Jensen LJ, Brunak S, "Mining electronic health records: towards better research applications and clinical care", Nat Rev Genet.2013, 14(1) pp.75.

5. L. Qiu, K. Gai, and M. Qiu, "Optimal big data sharing approach for tele-health in cloud computing", in Smart Cloud (Smart Cloud), IEEE International Conference on. IEEE, 2016, pp. 184189.

6. Siwei Lai, Xu Kang Liu, Jun Zhao, "Recurrent Convolutional Neural Networks for Text Classification", in proceeding of the twenty-ninth AAAI Conference on Artificial Intelligence 2015.

7. Xingyou Wang, Weijie Jiang, Zhiyong Luo, "Combination of Convolutional and Recurrent Neural Network for Sentimental Analysis of 
Short Texts", International Conference on Computational Linguistics: technical papers, 2016, pg 2428-2437

8. Dipak V. Patil, R.S. Bichkar, "Multiple Imputation of Missing Data with Genetic Algorithm based Techniques", IJCA Special issue on Evolutionary Computation for Optimization Technique, 2010.

9. Ying Wen, Weinan Zhang, Rui Luo, Jun Wang, "Learning text representation using recurrent convolutional neural network with highway letters", NeuIR 16 SIGIR Workshop on Neural Information Retrieval, July 21, 2016, Pisa, Italy.

10. N. Nori, H. Kashima, K. Yamashita, H. Ikai, and Y. Imanaka, "Simultaneous modeling of multiple diseases for mortality prediction in acute hospital care", in Proceedings of the 21th ACM SIGKDD International Conference on Knowledge Discovery and Data Mining. ACM, 2015.

11. J. C. Ho, C. H. Lee, and J. Ghosh, "Septic shock prediction for patients with missing data", ACM Transactions on Management Information Systems (TMIS), 2014 , vol. 5, no. 1, p. 1.

12. https://en.wikipedia.org/wiki/Recurrent neural network.

13. Kanchan M. Tarwani, Swathi Edem, "Survey on Recurrent Neural Network in Natural Language Processing", International Journal of Engineering Trends and Technology (IJETT), Volume 48 Number 6, June 2017.

14. JanKoutnik, Klaus Greff, Faustino Gomez, Jurgen Schmidhuber, "A Clockwork RNN", IDSIA, USI SUPSI, Manno Lugano, CH-6928, Switzerland.

15. R.Vijaya Kumar Reddy, K. Prudvi Raju, M. Jogendra Kumar, CH. Sujatha, P. Ravi Prakash, "Prediction of Heart Disease Using Decision Tree Aproach", International Journal of Advanced Research in Computer Science and Software Engineering, Volume 6, Issue 3, March 2016.

16. NetworksDimitrios H. Mantzaris, George C. Anastassopoulos and Dimitrios K. Lymberopoulos, "Medical Disease Prediction Using Artificial Neural", BioInformatics and BioEngineering, 2008. BIBE 2008. 8th IEEE International Conference on 08 December 2008.

17. Youn-Jung Son, Hong-Gee Kim, Eung-Hee Kim, Sangsup Choi, and Soo-Kyoung Lee, "Application of Support Vector Machine for Prediction of Medication Adherence in Heart Failure Patients", Health Information Research, v.16, 2010.

18. AlaaElsayad and Mahmoud Fakhr, "Diagnosis of Cardiovascular Diseases with Bayesian
Classifiers", Department of Computers and Systems, Electronics Research Institute, 12622 Bohoth St., Dokki, Geza, Egypt, 2015.

19. M. Akhil Jabbar, Dr. Priti Chandra, Dr. B.L Deekshatulu, Heart Disease Prediction System using Associative Classification and Genetic Algorithm", International Conference on Emerging Trends in Electrical, Electronics and Communication Technologies, -ICECIT, 2012.

20. K.B., Prakash. "Information extraction in current Indian web documents." International Journal of and Technology(UAE), 2018: 68-71.

21. Kolla B.P., Dorairangaswamy M.A., Rajaraman A. "A neuron model for documents containing multilingual Indian texts." 2010 International Conference on Computer and Communication Technology, ICCCT-2010,2010: 451-454.

22. Kolla B.P., Raman A.R. "Data Engineered Content Extraction Studies for Indian Web Pages." Advances in Intelligent Systems and Computing, 2019: 505-512.

23. Prakash K.B., Dorai Rangaswamy M.A. "Content extraction studies using neural network and attribute generation." Indian Journal of Science and Technology, 2016: 1-10.

24. Prakash K.B., Dorai Rangaswamy M.A., Raman A.R. "Text studies towards multi-lingual content mining for web communication." Proceedings of the 2nd International Conference on Trendz in Information Sciences and Computing, TISC-2010, 2010: 28-31.

25. Prakash K.B., Rangaswamy M.A.D. "Content extraction of biological datasets using soft computing techniques.", Journal of Medical Imaging and Health Informatics, 2016: 932-936.

26. H.I. Okagbue, N.J. Peter, A.O. Akinola, C.O. Iroham, A.P. Opoko, "Smart Review of the Application of Genetic Algorithm in Construction and Housing", International Journal of Advanced Trends in Computer Science and Engineering, Vol 9(1) (2020), pp: 266-273, DoI: https://doi.org/10.30534/ijatcse/2020/ 40912020.

27. Bandara, Ravimal. "Nature Inspired Dimensional Reduction Technique for Fast and Invariant Visual Feature Extraction." International Journal of Advanced Trends in Computer Science and Engineering, Vol 8(3) (2019), pp: 696-706. DoI: 10.30534/ijatcse/2019/57832019

28. Bharat, Vinod \& Malik, Dr. Study of Detection of Various types of Cancers by using Deep Learning: A Survey. International Journal of Advanced Trends in Computer Science and Engineering, Vol 8, ,2019, pp: 1228-1233, DoI: 10.30534/ ijatcse/2019/31842019. 